\title{
Study on Data Synchronization Technology of Non-contact Coordinate Measurement Machine
}

\author{
Jingliang Liu", a, Yi Chao, ${ }^{2, b}$, and Xue $\mathrm{Hao}^{3, \mathrm{c}}$ \\ ${ }^{1,2,3}$ Aviation Key Laboratory of Science and Technology on Precision Manufacturing, \\ NanYuan East Road NO.5,FengTai District, BeiJing,China

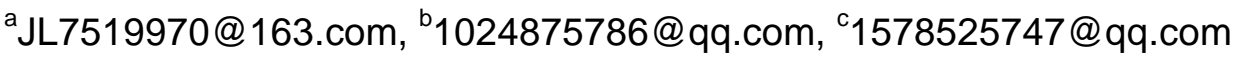

\begin{abstract}
Keywords: Data Synchronization, Non-contact Measurement, Optical probe
Abstract. This paper analyzes the current situation of non-contact measurement at home and abroad, a profound understanding of the importance of data synchronous of non-contact measuring machine. Then, design three types of data synchronization experiment, and set up verification environments respectively to analyze the character of data synchronization in non-contact measurement systems. Finally, sending synchronous pulse by hardware can realize the data synchronization of non-contact coordinate measurement machine.
\end{abstract}

\section{Introduction}

In the field of precision measurement, more than $95 \%$ of coordinate measuring machines(CMM) are using contact probes ${ }^{[1]}$. However, with the development of technology, the detection efficiency of contact measuring machines is more and more unable to meet the needs of efficient and precise detection. At the same time, the coordinate measuring machine based on optical noncontact probe has developed rapidly. WENZEL and Hexagon have introduced the mature non-contact measuring machine products, and the efficiency of detection is ensuring, and the detection accuracy is gradually improving, and their products are gradually recognized by the CMM market. But the domestic technologies of CMM are developing slowly in the field of non-contact measurement. The reason is that many key technologies of the non-contact measuring machine have not been solved. Among the key technologies, the non-contact measurement data synchronization is the one need to be solved immediately and rapidly.

Beijing Precision Engineering Institute for Aircraft Industry has rich experience in the field of coordinate measurement machine. After many experiments of technological studies, we have grasped the synchronization technology of optical probe data and achieved significant breakthroughs.

\section{Data Synchronization Technology}

In order to achieve efficient measurement, The measurement method of non-contact measuring machine is scanning measurement. In non-contact scanning process, the most critical technology is data synchronization between optical probe and grating data of coordinate measuring machine. The methods of data synchronization include software synchronization, hardware synchronization, and pulse synchronization.

\section{Data Synchronization by Advantec 1784U Card}

Based on VS2010, PC software is developed, and the data acquisition software of optical probe measurement and coordinate measuring machine is programmed. The test results of the detection device are collected at the same time by the software level. The data acquisition flow chart is as follows: 


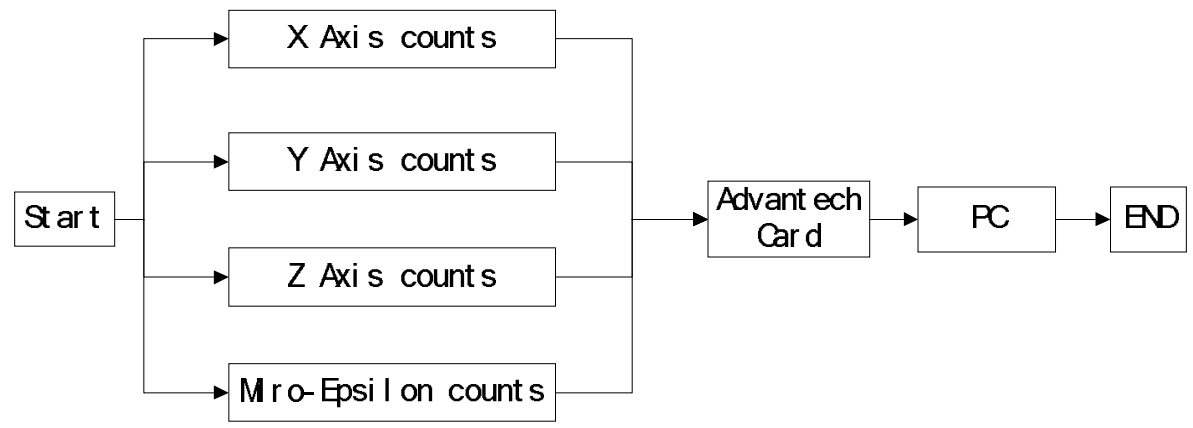

Fig.1 Flow Chart by Advantec 1784U Card

\section{Data Synchronization by PMAC Multi-Functional Card}

The position information of the coordinate measuring machine and the measured value of the optical probe are obtained by collecting the four $\pm 10 \mathrm{~V}$ analog quantities of the PMAC board. The system architecture of data synchronization test is as follows.

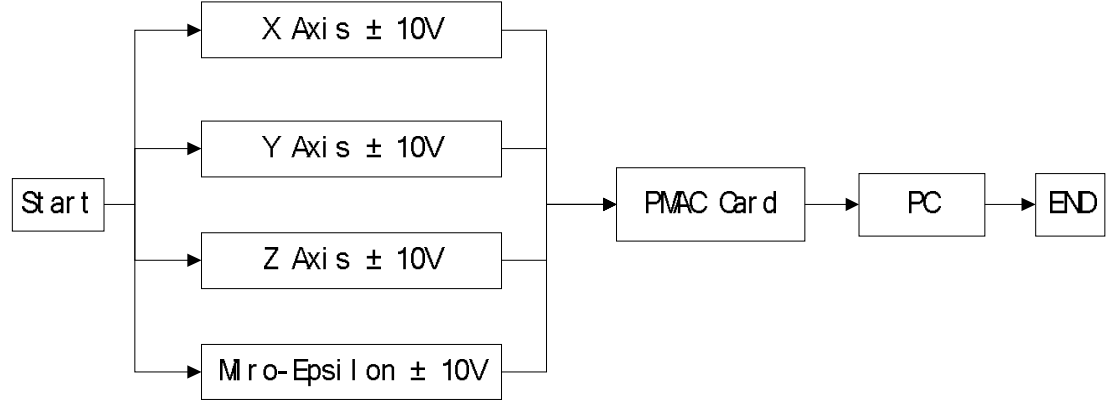

Fig2. Flow Chart by PMAC Card

\section{Data Synchronization by Hardware Pulse}

Using the AC6624 card and designing the synchronous circuit, the synchronous pulse is sent to the motion controller and laser measurement of the coordinate measuring machine at the same time, and then the $\mathrm{CMM}^{\prime}$ s three axis value and the laser probe measurement value are recorded. In the design of the synchronous pulse circuit, the two synchronous pulses must be separated from the original signal to prevent interference between the pulses. The system block diagram is as follows:

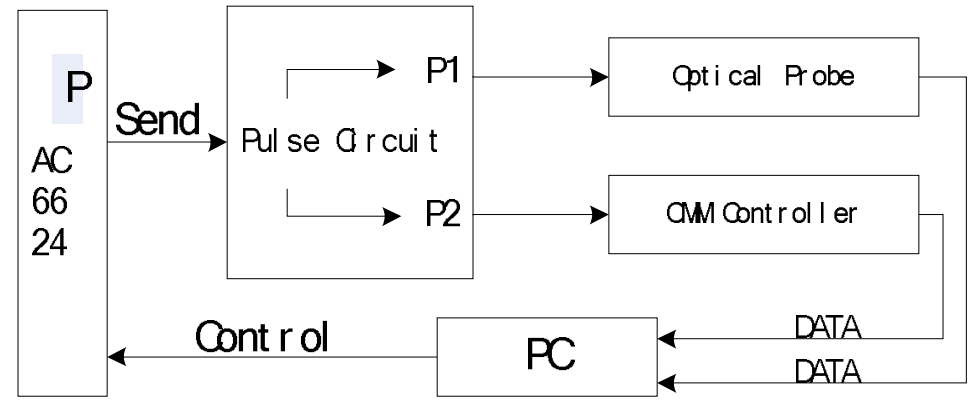

Fig.3 Flow Chart by Hardware Pulse

\section{The Introduction of Experiment System}

The data synchronization experiment environment of the non-contact measuring machine includes the following parts:

1 ) CMM: Pearl-OSM, the accuracy is $2.5+\mathrm{L} / 350$ um after compensation ${ }^{[3]}$;

2 ) No-contact Probe: ILD1700-20, Micro-Epsilon, German. The distance of measurement is from $40 \mathrm{~mm}$ to $60 \mathrm{~mm}^{[2]}$;

3 ) Advantech Card: 1784U ;

4 ) PMAC Multi-Functional Card ; 
5 ) AC6624 Multi-Functional Card ;

6 ) Standard Ball: the diameter is $49.985 \mathrm{~mm}$ 。

The scanning measurement synchronization test system of the non-contact coordinate measurement machine is shown in the following diagram.

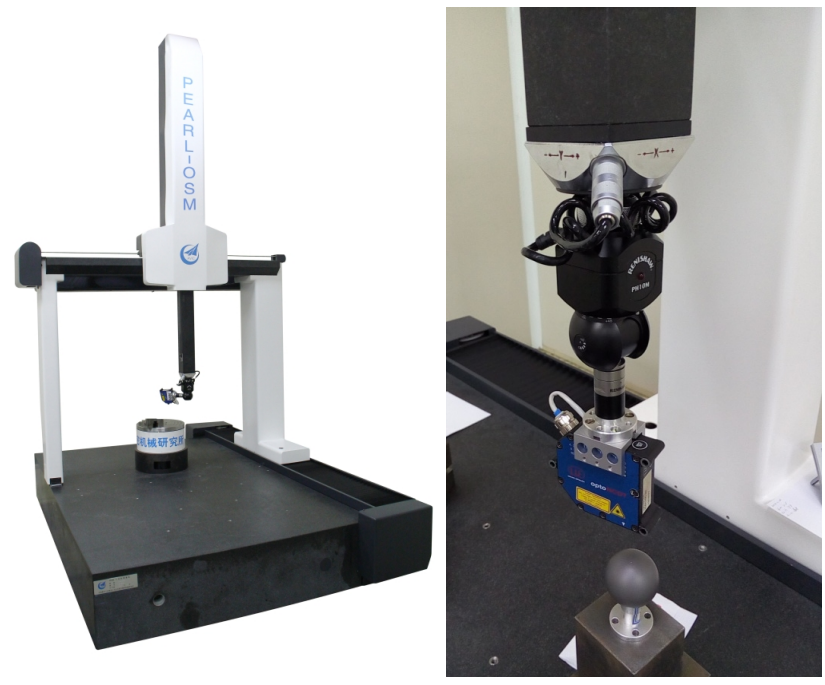

Fig. 4 Non-contact Measurement System

\section{Test}

The test process of the data Synchronization of the non-contact CMM is as follows:

1) Put the standard ball in the workspace of CMM, and then control the CMM to move from A to $\mathrm{B}$ ( $\mathrm{A}$ and $\mathrm{B}$ are $3 \mathrm{D}$ points in the $\mathrm{CMM}$ workspace), and the measurement values of the measuring machine's $\mathrm{XYZ}$ axis and the optical probe during the movement are recorded.

2) According to the recorded measured values of the $X Y Z$ axis of the coordinate measuring machine, then control the measuring machine move to the same position and the value of the optical probe is recorded again.

3) Compare the value of probe measurement in the first and second steps.

\section{Data Synchronization by Advantec 1784U Card}

According to synchronous data experiment process, the results of the data synchronous measurement test are shown in the following table.

Table 1 Test Record Base on the Advantec 1784U

\begin{tabular}{|c|c|c|c|c|c|c|}
\hline No. & $\mathrm{X}(\mathrm{mm})$ & $\mathrm{Y}(\mathrm{mm})$ & $\mathrm{Z}(\mathrm{mm})$ & $\mathrm{LS}(\mathrm{mm})$ & $\mathrm{LD}(\mathrm{mm})$ & $\mathrm{E}(\mathrm{mm})$ \\
\hline 1 & 534.1195 & 298.363 & -513.82 & 45.7612 & 45.8070 & 0.0458 \\
\hline 2 & 534.119 & 297.6145 & -513.821 & 45.4895 & 45.6464 & 0.1569 \\
\hline 3 & 534.1175 & 296.8145 & -513.82 & 45.2128 & 45.1704 & -0.0424 \\
\hline 4 & 534.12 & 295.6135 & -513.82 & 44.8501 & 44.9550 & 0.1049 \\
\hline 5 & 534.1195 & 294.1615 & -513.82 & 44.4949 & 44.5983 & 0.1034 \\
\hline 6 & 534.118 & 292.9615 & -513.82 & 44.2669 & 44.0824 & -0.1845 \\
\hline 7 & 534.1185 & 291.4125 & -513.82 & 44.0562 & 43.9420 & -0.1142 \\
\hline 8 & 534.119 & 289.265 & -513.82 & 43.9316 & 44.0903 & 0.1587 \\
\hline 9 & 534.1195 & 287.7625 & -513.82 & 43.959 & 43.8946 & -0.0644 \\
\hline 10 & 534.118 & 286.015 & -513.82 & 44.0986 & 44.0711 & -0.0275 \\
\hline
\end{tabular}

\section{Data Synchronization by PMAC Multi-Functional Card}

According to synchronous data experiment process, the results of the data synchronous measurement test are shown in the following table. 
Table 2 Test Record Base on the PMAC Card

\begin{tabular}{|c|c|c|c|c|c|c|}
\hline No. & $\mathrm{X}(\mathrm{mm})$ & $\mathrm{Y}(\mathrm{mm})$ & $\mathrm{Z}(\mathrm{mm})$ & $\mathrm{LS}(\mathrm{mm})$ & $\mathrm{LD}(\mathrm{mm})$ & $\mathrm{E}(\mathrm{mm})$ \\
\hline 1 & 534.1195 & 298.363 & -513.82 & 45.7612 & 45.7435 & -0.0177 \\
\hline 2 & 534.119 & 297.6145 & -513.821 & 45.4895 & 45.4110 & -0.0785 \\
\hline 3 & 534.1175 & 296.8145 & -513.82 & 45.2128 & 45.2578 & 0.0450 \\
\hline 4 & 534.12 & 295.6135 & -513.82 & 44.8501 & 44.8882 & 0.0381 \\
\hline 5 & 534.1195 & 294.1615 & -513.82 & 44.4949 & 44.5845 & 0.0896 \\
\hline 6 & 534.118 & 292.9615 & -513.82 & 44.2669 & 44.2742 & 0.0073 \\
\hline 7 & 534.1185 & 291.4125 & -513.82 & 44.0562 & 44.0911 & 0.0349 \\
\hline 8 & 534.119 & 289.265 & -513.82 & 43.9316 & 43.9685 & 0.0369 \\
\hline 9 & 534.1195 & 287.7625 & -513.82 & 43.959 & 43.9249 & -0.0341 \\
\hline 10 & 534.118 & 286.015 & -513.82 & 44.0986 & 44.1408 & 0.0422 \\
\hline
\end{tabular}

\section{Data Synchronization by Hardware Pulse}

According to synchronous data experiment process, the results of the data synchronous measurement test are shown in the following table.

Table 3 Test Record Base on the Hardware Pulse

\begin{tabular}{|c|c|c|c|c|c|c|}
\hline No. & $\mathrm{X}(\mathrm{mm})$ & $\mathrm{Y}(\mathrm{mm})$ & $\mathrm{Z}(\mathrm{mm})$ & $\mathrm{LS}(\mathrm{mm})$ & $\mathrm{LD}(\mathrm{mm})$ & $\mathrm{E}(\mathrm{mm})$ \\
\hline 1 & 534.1195 & 298.363 & -513.82 & 45.7612 & 45.7632 & 0.0020 \\
\hline 2 & 534.119 & 297.6145 & -513.821 & 45.4895 & 45.4922 & 0.0027 \\
\hline 3 & 534.1175 & 296.8145 & -513.82 & 45.2128 & 45.2138 & 0.0010 \\
\hline 4 & 534.12 & 295.6135 & -513.82 & 44.8501 & 44.8501 & 0.0000 \\
\hline 5 & 534.1195 & 294.1615 & -513.82 & 44.4949 & 44.4935 & -0.0014 \\
\hline 6 & 534.118 & 292.9615 & -513.82 & 44.2669 & 44.2646 & -0.0023 \\
\hline 7 & 534.1185 & 291.4125 & -513.82 & 44.0562 & 44.0541 & -0.0021 \\
\hline 8 & 534.119 & 289.265 & -513.82 & 43.9316 & 43.9293 & -0.0023 \\
\hline 9 & 534.1195 & 287.7625 & -513.82 & 43.959 & 43.9597 & 0.0007 \\
\hline 10 & 534.118 & 286.015 & -513.82 & 44.0986 & 44.0962 & -0.0024 \\
\hline
\end{tabular}

Note: " $\mathrm{X}$ " means $\mathrm{X}$ axis measurement value; "Y" means $\mathrm{Y}$ axis measurement value; " $\mathrm{Z}$ " means $\mathrm{Z}$ axis measurement value; "LS" means the optical probe measurement values while the probe weas scanning; "LD" optical probe measurement record values; "E" means the difference value of "LS" and "LD".

From the three tables above, the synchronization error of Advantech $1784 \mathrm{U}$ is about $0.1 \mathrm{~mm}$, the synchronization error of PMAC is about $0.04 \mathrm{~mm}$, and the synchronization error of the hardware pulse is about $0.002 \mathrm{~mm}$.

visible hardware synchronous pulse mode can realize three axis measuring three coordinate values of synchronous measurement and optical probe values. To meet the need of precise measurement.

\section{Summary}

This paper has analyzed the data synchronization in non-contact measurement system by design three types of expriments. What's more, in order to obtain the synchronous characteristics, three types of experiments were constructed and tested many times at the same situation. At the end of the day, the mode of hardware pulse is used for data synchronization.

\section{Acknowledgements}

This work was financially supported by the AVIC Inovation Foundation (2014F30334).

\section{References}

[1] Guoxiong Zhang, The Coordinate Measurement Machine, Tianjin,1999.

[2] Information on http:www.micro-epsilon.com

[3] Information on http:www.Pantec.com 